\title{
Comparison of Iterative and Non-Iterative Strain-Gage Balance Load Calculation Methods
}

\author{
N. Ulbrich* \\ Jacobs Technology Inc., Moffett Field, California 94035-1000
}

The accuracy of iterative and non-iterative strain-gage balance load calculation methods was compared using data from the calibration of a force balance. Two iterative and one non-iterative method were investigated. In addition, transformations were applied to balance loads in order to process the calibration data in both direct-read and force balance format. NASA's regression model optimization tool BALFIT was used to generate optimized regression models of the calibration data for each of the three load calculation methods. This approach made sure that the selected regression models met strict statistical quality requirements. The comparison of the standard deviation of the load residuals showed that the first iterative method may be applied to data in both the direct-read and force balance format. The second iterative method, on the other hand, implicitly assumes that the primary gage sensitivities of all balance gages exist. Therefore, the second iterative method only works if the given balance data is processed in force balance format. The calibration data set was also processed using the non-iterative method. Standard deviations of the load residuals for the three load calculation methods were compared. Overall, the standard deviations show very good agreement. The load prediction accuracies of the three methods appear to be compatible as long as regression models used to analyze the calibration data meet strict statistical quality requirements. Recent improvements of the regression model optimization tool BALFIT are also discussed in the paper.

\section{Nomenclature}

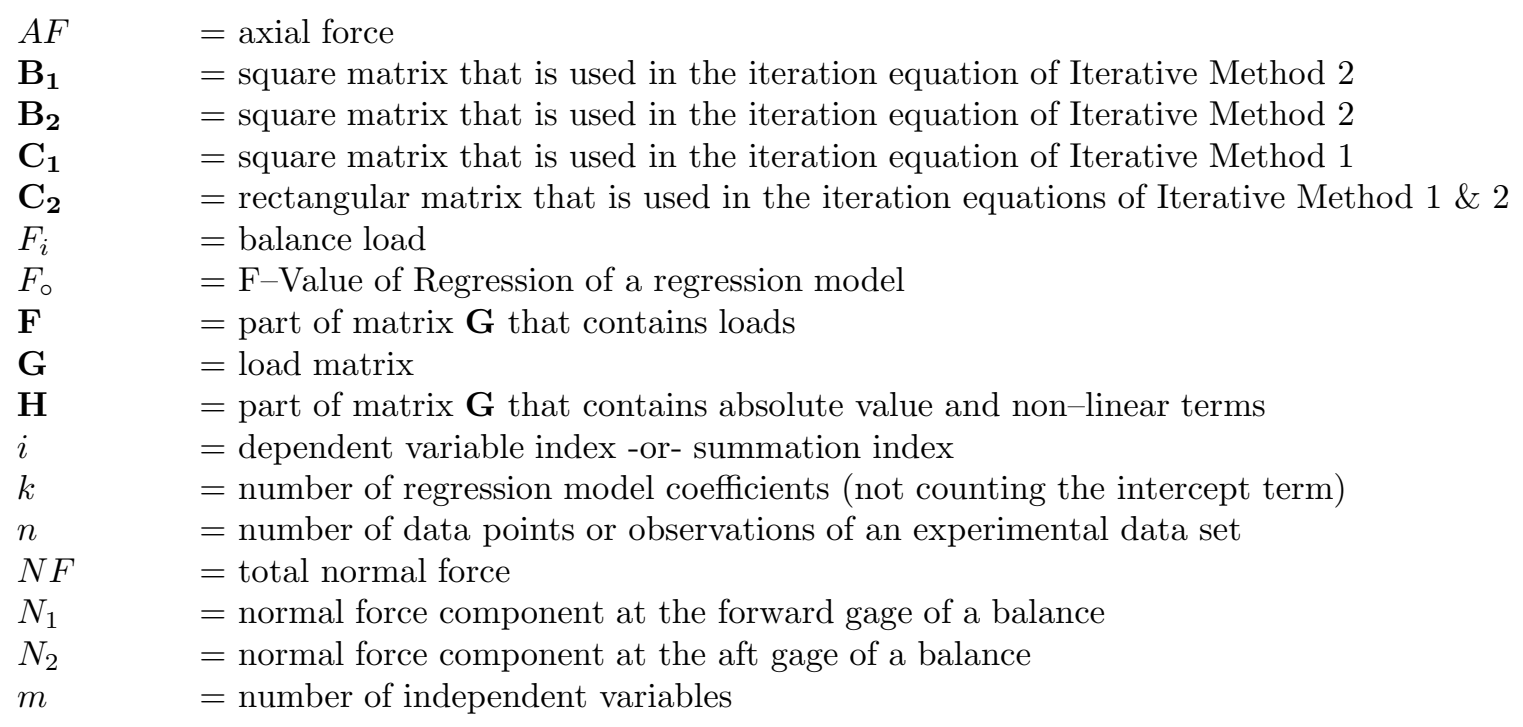

\footnotetext{
* Aerodynamicist; Jacobs Technology Inc. Copyright $(2010$ by the American Institute of Aeronautics and Astronautics, Inc. - The U.S. Government has a royalty-free license to exercise all rights under the copyright claimed herein for Governmental purposes. All other rights are reserved by the copyright owner.
} 


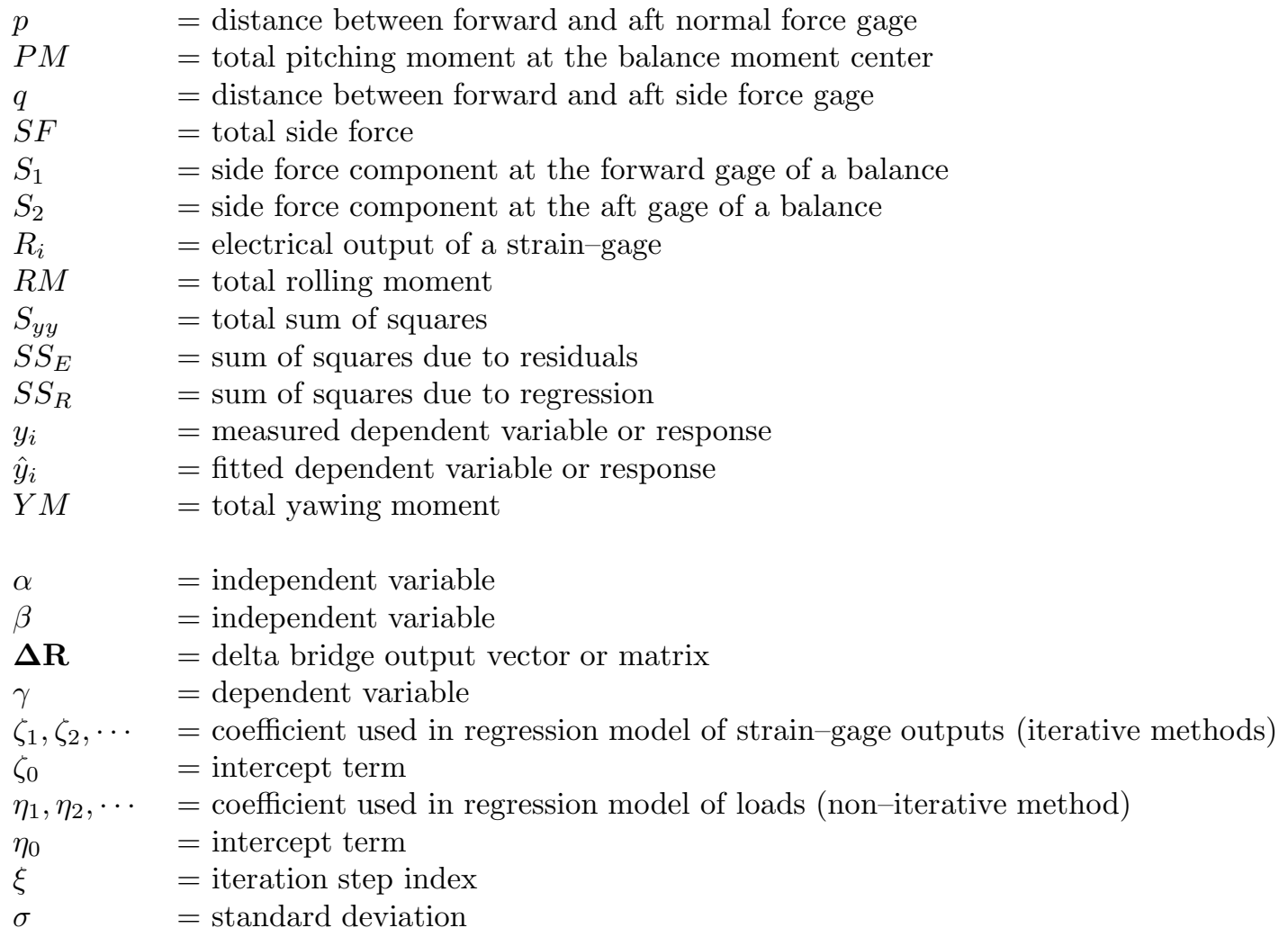

\section{Introduction}

Different methods are used in wind tunnel testing in order to compute balance loads from measured strain-gage outputs. These methods may be separated into two classes: iterative and non-iterative methods. In principle, both classes of methods fit a given set of balance calibration data in the least squares sense and use the result of the regression analysis for the prediction of balance loads during a wind tunnel test. The two classes of methods, however, differ in the selection of the independent and dependent variables that are used for the regression analysis of the balance calibration data.

Two iterative methods (method 1 and method 2) are currently being used in the wind tunnel testing community. These iterative methods fit the gage outputs as a function of the applied calibration loads and use an iteration equation in order to predict loads from gage outputs. The non-iterative method, on the other hand, directly fits the balance calibration loads as a function of the measured gage outputs. Table 1 summarizes basic differences between the regression analysis of balance calibration data using iterative and non-iterative methods.

Table 1: Differences between strain-gage balance calibration analysis methods.

\begin{tabular}{|c|c|c|}
\hline & $\begin{array}{c}\text { ITERATIVE METHODS } \\
\text { (METHOD 1 \& METHOD 2) }\end{array}$ & $\begin{array}{c}\text { NON-ITERATIVE METHOD } \\
\text { (DIRECT-COMPUTE METHOD) }\end{array}$ \\
\hline \hline INDEPENDENT VARIABLE & LOADS & GAGE OUTPUTS \\
\hline DEPENDENT VARIABLE & GAGE OUTPUTS & LOADS \\
\hline
\end{tabular}

It is often unclear how the choice of the balance calibration analysis method, i.e., the choice of the load calculation method, influences the load prediction accuracy of the balance. In addition, the processing of data in direct-read or force/moment balance format may also have an influence on the load prediction accuracy. Therefore, it was decided to perform a study that would use a realistic balance calibration data set in order find answers to some of these questions. Results of this investigation are discussed in great detail 
in the present paper.

During the study three load calculation methods were applied to balance calibration data that was given in two load formats. Therefore, a total of six independent regression analysis cases were investigated. The three different load calculation methods are explained in some detail in the next section of the paper. Then, calibration data used in the present study is described and final results of the load prediction study are presented and compared. Some recent improvements of a regression model optimization process are also discussed as this optimization process was used to obtain regression models of the calibration data for the current study.

\section{Description of Load Calculation Methods}

In general, iterative methods use a two-step approach in order to determine loads from measured gage outputs. First, electrical outputs of balance strain-gages are fitted as a function of calibration loads. Then, the loads are computed using an iteration equation that is derived from the global regression analysis of the strain-gage outputs. The balance loads are the independent variables of the regression analysis problem. The electrical outputs of the gages, on the other hand, are the dependent variables. Therefore, the regression model of the gage outputs can be expressed as follows:

$$
R_{i}=\zeta_{0}(i)+\zeta_{1}(i) \cdot F_{1}+\zeta_{2}(i) \cdot F_{2}+\cdots+\zeta_{m}(i) \cdot F_{m}+\cdots
$$

Detailed explanations of iterative methods are given in Refs. [1], [2], and [3]. For the present study two iterative methods, i.e., Iterative Method 1 and Iterative Method 2 were selected. Iterative Method 1 is described in great detail in Ref. [1]. Iterative Method 2 is explained in Ref. [3]. Figure 1 compares the definition and initial guess of the iteration equations that the two iterative methods use (from Ref. [3]).

The Non-Iterative Method exchanges the independent and dependent variables that Iterative Method 1 and Iterative Method 2 use. Now, the strain-gage outputs become independent variables and the balance loads are the dependent variables for the regression analysis of the balance calibration data. Therefore, the balance loads are fitted as a function of the measured strain-gage outputs.

$$
F_{i}=\eta_{0}(i)+\eta_{1}(i) \cdot R_{1}+\eta_{2}(i) \cdot R_{2}+\cdots+\eta_{m}(i) \cdot R_{m}+\cdots
$$

The Non-Iterative Method has the advantage that it is a one-step method. No iteration is needed in order to compute loads from measured strain-gage outputs during a wind tunnel test. An analyst, however, must not forget that the method ignores the fact that the balance loads are the "true" independent variables of the calibration experiment as loads are "applied" and strain-gage outputs are "measured" during the calibration of a balance. Therefore, the success of the Non-Iterative Method depends on the fundamental assumption that an exchange of the independent and dependent variables of the calibration data set does not negatively influence the mathematical description of the "true" physical behavior of the balance.

\section{Description of Balance Calibration Data}

Machine calibration data of NASA's MC60D balance was selected for the present study. The balance is shown in Fig. 2. It is a force balance that was manufactured by Triumph/Force Measurement Systems in 2008. Table 2 lists load capacities of the balance in direct-read format:

Table 2: Load capacities of the MC60D balance in direct-read format.

\begin{tabular}{|l|c|c|c|c|c|c|}
\hline & $N F, \mathrm{lbs}$ & $P M$, in-lbs & $S F, \mathrm{lbs}$ & $Y M$, in-lbs & $R M$, in-lbs & $A F, \mathrm{lbs}$ \\
\hline CAPACITY & 5000 & 20000 & 2500 & 10000 & 5000 & 700 \\
\hline
\end{tabular}

The balance calibration data was obtained in Triumph's balance calibration machine (ABCS). The supplied machine calibration data of the balance was already corrected for the weight of the balance shell and other calibration fixtures. This characteristic was an advantage for the current study as the fitted 
balance loads were not influenced by tare load estimate differences that may be caused by small numerical differences between the selected load calculation methods.

In general, it is important to assess the accuracy of a load calculation method at (i) data points that are used to develop the regression model of the balance characteristics and at (ii) confirmation points that are independent of the regression model. Therefore, it was decided to split the original machine calibration data set of the MC60D balance into two subsets. The first subset (subset 1) was used to develop the regression models for the three load calculation methods. The second subset (subset 2) contained confirmation points. Table 3 below summarizes important characteristics of the balance and the calibration data set that was used for the present study.

Table 3: Balance and calibration data set characteristics of the MC60D balance.

\begin{tabular}{|c|c|}
\hline BALANCE NAME & MC-60H-2.00D \\
\hline BALANCE TYPE & FORCE BALANCE \\
\hline DIAMETER & $2.0[\mathrm{in}]$ \\
\hline GAGE DISTANCE $(p, q)$ & DECEMBER 2008 \\
\hline CALIBRATION DATE & MACHINE CALIBRATION \\
\hline CALIBRATION METHOD & 1906 \\
\hline TOTAL NUMBER OF CALIBRATION POINTS & 953 \\
\hline NUMBER OF DATA POINTS & 953 \\
\hline NUMBER OF CONFIRMATION POINTS & TARE CORRECTED LOADS \\
\hline LOAD FORMAT & GAGE OUTPUT DIFFERENCES \\
\hline GAGE OUTPUT FORMAT &
\end{tabular}

Balance load residuals are defined as the difference between the measured and fitted (predicted) loads. Standard deviations of the residuals of both subsets were computed in order to compare the predictive capability of the three load calculation methods. In addition, the load capacities listed in Table 2 were used to express the standard deviation of the residuals in units of percent whenever data was processed in direct-read format.

\section{Regression Model Optimization Process}

It was decided to use only regression models of the calibration data for the current study that meet strict statistical quality requirements. These regression models were identified using a regression model optimization process that was implemented in a regression analysis tool called BALFIT (see Refs. [2], [3], and [4] for a description of BALFIT's optimization process).

BALFIT uses a candidate math model search algorithm during the optimization of the regression model of a given set of multivariate experimental data. This optimized regression model is called "recommended math model." It is used for the final regression analysis of the experimental data set. In principle, the search algorithm minimizes a search metric by testing only those math models that (i) do not have linear or near-linear dependencies between terms and that (ii) satisfy a set of search constraints. The search metric, for example, may be the standard deviation of the PRESS residuals of the dependent variable of the regression analysis problem (see Ref. [5] for a description of different search metrics). Figure 3 summarizes basic steps of the latest version of the candidate math model search algorithm that is used to obtain the recommended math model. Different user choices (inputs) are listed on the left hand side of the flow chart that is depicted in Fig. 3. Each user choice influences the search process and the final term combination that the recommended math model will have.

Two significant improvements of BALFIT's candidate math model search algorithm were made in 2010. The first improvement is related to different search strategies that may be chosen during the regression model search. Now, an analyst may use forward selection or backward elimination as a search strategy. Table 4 
below compares advantages and disadvantages of the two search strategy choices:

Table 4: Search Strategy Options.

\begin{tabular}{|c|c|c|}
\hline & ADVANTAGES & DISADVANTAGES \\
\hline $\begin{array}{c}\text { FORWARD } \\
\text { SELECTION }\end{array}$ & $\bullet$ Constraints can be enforced simultaneously & $\begin{array}{l}\bullet \text { Benefit of term combinations may be overlooked } \\
\text { BACKWARD }\end{array}$ \\
\hline ELIMINATION & $\bullet$ Benefit of term combinations is recognized in every step & $\bullet$ Constraints cannot be enforced simultaneously \\
\hline
\end{tabular}

The primary search strategy, i.e., forward selection, has the advantage that both threshold based search constraints (constraint $2 \& 3$ in Fig. 3 ) can be enforced simultaneously (i.e, in parallel). Forward selection, however, may overlook benefits of certain term combinations as the regression model is built by adding terms during the search. Forward selection is also only suited for an automated search. The initial number of required math model tests is simply too large for a visual inspection of different statistical quality metrics that may be used to interactively select terms of the regression model.

The alternate search strategy, i.e., backward elimination, recognizes the benefit of math term combinations during every step of the search as the regression model is built by removing terms during the search. Backward elimination, however, cannot enforce the threshold based search constraints (constraint $2 \& 3$ in Fig. 3) simultaneously as a term selected for removal may not necessarily violate all constraints. Therefore, it was decided to implement the search constraints in "series" if backward elimination is selected as the search strategy. This implementation works in three steps. First, a regression model is tested using search constraint 2 (near-linear dependency test) with a temporarily elevated threshold of 50 . This test has priority over search constraint 3 (test of the significance of a term) until search constraint 2 is no longer violated during the search process. Then, search constraint 3 takes over. Now, search constraint 3 has priority over search constraint 2. Finally, search constraint 2 takes over again using the thresholds of 5 (or 10) after search constraint 3 can no longer be violated during the search process.

A second improvement was also implemented in the latest version of the candidate math model search algorithm. Previously, only the minimum of the search metric was used to identify the recommended math model after the completion of the candidate math model search. Now, it is also possible to use the maximum of the F-Value of Regression of the candidate math models for this purpose. Table 5 below lists the two identification metrics that an analyst may choose to identify the recommended math model after the completion of the candidate math model search.

Table 5: Identification Metric Options.

\begin{tabular}{|c|c|}
\hline & DESCRIPTION \\
\hline PRIMARY METRIC & MINIMUM OF THE SEARCH METRIC \\
\hline ALTERNATE METRIC & MAXIMUM OF THE F-VALUE OF REGRESSION \\
\hline
\end{tabular}

The minimum of the search metric is still used as the default choice for the identification of the recommended math model. The new identification metric, i.e., the maximum of the F-Value of Regression of the candidate math models, may also be selected. This alternate metric usually recommends a math model that has fewer terms than the math model that the primary identification metric suggests. A more detailed discussion of the new alternate identification metric is given in the appendix of the paper.

Experience with different types of experimental data sets has shown that it is important for a user of BALFIT's regression model optimization process to remember that different combinations

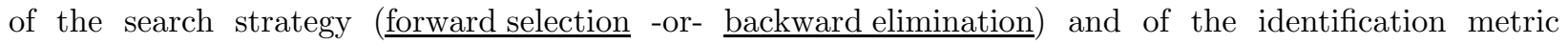
(minimum of the search metric -or- maximum of the F-Value of Regression) may have to be tested in order to find a regression model that meets an analyst's expectations. For the current study, however, it was decided to always use backward elimination and the minimum of the search metric for the optimization of a regression model as this combination lead to reasonable optimization results in all cases. 


\section{Analysis in Direct-Read Format}

During the first part of the study the calibration data of the MC60D balance was processed in directread format. Therefore, the balance loads were used in the following format: NF, PM, SF, YM, RM, and AF. The strain-gage outputs, on the other hand, remained unchanged. Input data files were prepared and calibration data points were processed using the three chosen load calculation methods. BALFIT's candidate math model search algorithm was applied in each case in order to obtain an optimized regression model of the data that met strict statistical quality requirements. Afterwards, load residuals and corresponding standard deviations were computed at the data points (subset 1) and the confirmation points (subset 2).

Table 6.1 and 6.2 below show the standard deviation of the load residuals of the calibration data points and the confirmation points for the three methods in both engineering units and percent of load capacity.

Table 6.1: Standard deviation of calibration data points (subset 1) in direct-read format.

\begin{tabular}{|c|c|c|c|c|c|c|}
\hline METHOD & $N F, \mathrm{lbs}$ & $P M$, in-lbs & $S F, \mathrm{lbs}$ & $Y M$, in-lbs & $R M$, in-lbs & $A F, \mathrm{lbs}$ \\
\hline Iterative 1 & $2.50(0.050 \%)$ & $9.61(0.048 \%)$ & $2.59(0.104 \%)$ & $11.56(0.116 \%)$ & $7.27(0.146 \%)$ & $0.43(0.061 \%)$ \\
Iterative 2 & diverg. & diverg. & diverg. & diverg. & diverg. & diverg. \\
Non-Iterative & $2.46(0.049 \%)$ & $9.62(0.048 \%)$ & $2.58(0.103 \%)$ & $11.87(0.119 \%)$ & $7.27(0.145 \%)$ & $0.43(0.061 \%)$ \\
\hline
\end{tabular}

Table 6.2: Standard deviation of confirmation points (subset 2) in direct-read format.

\begin{tabular}{|c|c|c|c|c|c|c|}
\hline METHOD & $N F, \mathrm{lbs}$ & $P M$, in-lbs & $S F, \mathrm{lbs}$ & $Y M$, in-lbs & $R M$, in-lbs & $A F, \mathrm{lbs}$ \\
\hline Iterative 1 & $2.46(0.049 \%)$ & $9.92(0.050 \%)$ & $2.65(0.106 \%)$ & $12.14(0.121 \%)$ & $7.44(0.149 \%)$ & $0.45(0.065 \%)$ \\
Iterative 2 & diverg. & diverg. & diverg. & diverg. & diverg. & diverg. \\
Non-Iterative & $2.45(0.049 \%)$ & $9.91(0.050 \%)$ & $2.60(0.104 \%)$ & $12.55(0.125 \%)$ & $7.55(0.151 \%)$ & $0.45(0.065 \%)$ \\
\hline
\end{tabular}

It is observed that the standard deviations of Iterative Method 1 and the Non-Iterative Method for the points of subset 1 and subset 2 show very good agreement. We also see that Iterative Method 2 did not converge. This behavior is directly linked to the existence of the primary gage sensitivities of the balance calibration data. Table 6.3 below shows the primary gage sensitivities of the balance in direct-read format.

Table 6.3: Primary gage sensitivities of balance calibration data in direct-read format.

\begin{tabular}{|c|c|c|c|c|c|c|}
\hline & $\frac{\partial N F}{\partial R_{1}}$ & $\frac{\partial P M}{\partial R_{2}}$ & $\frac{\partial S F}{\partial R_{3}}$ & $\frac{\partial Y M}{\partial R_{4}}$ & $\frac{\partial R M}{\partial R_{5}}$ & $\frac{\partial A F}{\partial R_{6}}$ \\
& {$\left[\frac{l b s}{\mu V / V}\right]$} & {$\left[\frac{i n-l b s}{\mu V / V}\right]$} & {$\left[\frac{l b s}{\mu V / V}\right]$} & {$\left[\frac{i n-l b s}{\mu V / V}\right]$} & {$\left[\frac{i n-l b s}{\mu V / V}\right]$} & {$\left[\frac{l b s}{\mu V / V}\right]$} \\
\hline SENSITIVITY & not defined & not defined & not defined & not defined & 3.7689 & 0.8330 \\
\hline
\end{tabular}

We see that the sensitivities of the first four load/gage output combinations are not defined. This observation explains the divergence of the load iteration scheme as Iterative Method 2 implicitly assumes that all primary gage sensitivities of the balance gages of the chosen load format exist (see Ref. [3] for a detailed description of Iterative Method 2).

\section{Analysis in Force Balance Format}

In the second part of the study the data of the MC60D was processed in force balance format as the balance gages behave like gages of a force balance. Therefore, the loads were converted to the following 
format: $N_{1}, N_{2}, S_{1}, S_{2}, R M$, and $A F$. The transformation equations are defined as follows (from Ref. [6]):

$$
\begin{aligned}
& N_{1}=\frac{N F}{2}+\frac{P M}{p} \\
& N_{2}=\frac{N F}{2}-\frac{P M}{p} \\
& S_{1}=\frac{S F}{2}+\frac{Y M}{q} \\
& S_{2}=\frac{S F}{2}-\frac{Y M}{q}
\end{aligned}
$$

where the distances $p$ and $q$ between the forward and aft gages of the normal and side force components are given as

$$
p=q=6.0[i n]
$$

The transformed calibration loads and the original gage outputs were processed using the three load calculation methods. Again, optimized regression models of the data were used for the analysis of the calibration data. Table 7.1 and 7.2 below show the standard deviation of the load residuals of the calibration data points (subset 1) and the confirmation points (subset 2) for the three methods in engineering units.

Table 7.1: Standard deviation of calibration data points (subset 1) in force balance format.

\begin{tabular}{|c|c|c|c|c|c|c|}
\hline METHOD & $N_{1}, \mathrm{lbs}$ & $N_{2}, \mathrm{lbs}$ & $S_{1}, \mathrm{lbs}$ & $S_{2}, \mathrm{lbs}$ & $R M$, in-lbs & $A F, \mathrm{lbs}$ \\
\hline Iterative 1 & 1.66 & 2.36 & 2.41 & 2.24 & 7.17 & 0.43 \\
Iterative 2 & 1.66 & 2.36 & 2.41 & 2.24 & 7.17 & 0.43 \\
Non-Iterative & 1.71 & 2.31 & 2.43 & 2.28 & 7.27 & 0.43 \\
\hline
\end{tabular}

Table 7.2: Standard deviation of confirmation points (subset 2) in force balance format.

\begin{tabular}{|c|c|c|c|c|c|c|}
\hline METHOD & $N_{1}, \mathrm{lbs}$ & $N_{2}, \mathrm{lbs}$ & $S_{1}, \mathrm{lbs}$ & $S_{2}, \mathrm{lbs}$ & $R M$, in-lbs & $A F, \mathrm{lbs}$ \\
\hline Iterative 1 & 1.66 & 2.41 & 2.52 & 2.33 & 7.33 & 0.45 \\
Iterative 2 & 1.66 & 2.41 & 2.52 & 2.33 & 7.33 & 0.45 \\
Non-Iterative & 1.72 & 2.38 & 2.54 & 2.36 & 7.55 & 0.45 \\
\hline
\end{tabular}

This time, the primary gage sensitivities of all balance gages are defined (see Table 7.3 below). Therefore, Iterative Method 2 also converged and corresponding balance load residuals were computed.

Table 7.3: Primary gage sensitivities of balance calibration data in force balance format.

\begin{tabular}{|c|c|c|c|c|c|c|}
\hline & $\frac{\partial N_{1}}{\partial R_{1}}$ & $\frac{\partial N_{2}}{\partial R_{2}}$ & $\frac{\partial S_{1}}{\partial R_{3}}$ & $\frac{\partial S_{2}}{\partial R_{4}}$ & $\frac{\partial R M}{\partial R_{5}}$ & $\frac{\partial A F}{\partial R_{6}}$ \\
& {$\left[\frac{l b s}{\mu V / V}\right]$} & {$\left[\frac{l b s}{\mu V / V}\right]$} & {$\left[\frac{l b s}{\mu V / V}\right]$} & {$\left[\frac{l b s}{\mu V / V}\right]$} & {$\left[\frac{i n-l b s}{\mu V / V}\right]$} & {$\left[\frac{l b s}{\mu V / V}\right]$} \\
\hline SENSITIVITY & 2.6396 & 2.1571 & 2.1668 & 2.1341 & 3.7689 & 0.8330 \\
\hline
\end{tabular}

Several observations can be made if results listed in Table 7.1 and 7.2 are compared: (1) all methods appear to have a similar load prediction accuracy as the standard deviations of the load residuals of both 
subsets show very good agreement; (2) the standard deviations of both iterative methods are identical. The second observation is expected as both iterative methods, by design, are developed from the same set of regression coefficients that were used to fit the data.

\section{Comparison of Results}

It would be interesting to directly compare results in force balance format (Table 7.1 and 7.2) with results in direct-read format (Table 6.1 and 6.2). This comparison would allow us to see if the load format selection influenced the load prediction accuracy. The comparison can be performed if the standard deviations reported in Table 7.1 and 7.2 are converted from force balance to direct-read format. Afterwards it is possible to express the converted values as a percentage of the balance load capacity which will simplify the comparison of the converted values with those values that are given in Table 6.1 and 6.2.

The format conversion equations for the standard deviation can be understood by discussing a simple example. Let us assume that variables $\alpha$ and $\beta$ are a set of two independent variables that describe the behavior of a dependent variable called $\gamma$. Then, we can write:

$$
\gamma=\gamma(\alpha, \beta)
$$

We know from calculus that the individual standard deviations of the independent variables are related to the standard deviation of the dependent variable using the following relationship:

$$
\sigma(\gamma) \approx \sqrt{\left[\frac{\partial \gamma}{\partial \alpha} \cdot \sigma(\alpha)\right]^{2}+\left[\frac{\partial \gamma}{\partial \beta} \cdot \sigma(\beta)\right]^{2}}
$$

We also know, after applying the classical strain-gage balance load transformation given in Ref. [6] to the balance loads, that the following relationship between the total normal force and its components is valid:

$$
N F=\gamma\left(N_{1}, N_{2}\right)=N_{1}+N_{2}
$$

For the total pitching moment at the balance moment center the following relationship applies:

$$
P M=\mu\left(N_{1}, N_{2}\right)=\left(N_{1}-N_{2}\right) \cdot p / 2
$$

Now, after applying Eq. (5b) to Eq. (6a), we get for the standard deviation of the total normal force the relationship:

$$
\sigma(N F) \approx \sqrt{\left[\sigma\left(N_{1}\right)\right]^{2}+\left[\sigma\left(N_{2}\right)\right]^{2}}
$$

Similarly, after applying Eq. (5b) to Eq. (6b), we get for the standard deviation of the total pitching moment the relationship:

$$
\sigma(P M) \approx \frac{p}{2} \cdot \sqrt{\left[\sigma\left(N_{1}\right)\right]^{2}+\left[\sigma\left(N_{2}\right)\right]^{2}}
$$

Equivalent relationships can be derived for $S F$ and $Y M$ using the side force components $S_{1}$ and $S_{2}$ as independent variables. Now, after converting the standard deviations given in Table 7.1 and 7.2 from force balance to direct-read format, we get the following results in engineering units and in percent of the load capacity:

Table 8.1: Converted standard deviation of calibration data points (subset 1).

\begin{tabular}{|c|c|c|c|c|c|c|}
\hline METHOD & $N F$, lbs & $P M$, in-lbs & $S F$, lbs & $Y M$, in-lbs & $R M$, in-lbs & $A F$, lbs \\
\hline Iterative 1 & $2.88(0.058 \%)$ & $8.65(0.043 \%)$ & $3.29(0.131 \%)$ & $9.87(0.099 \%)$ & $7.17(0.143 \%)$ & $0.43(0.061 \%)$ \\
Iterative 2 & $2.88(0.058 \%)$ & $8.65(0.043 \%)$ & $3.29(0.131 \%)$ & $9.87(0.099 \%)$ & $7.17(0.143 \%)$ & $0.43(0.061 \%)$ \\
Non-Iterative & $2.87(0.057 \%)$ & $8.62(0.043 \%)$ & $3.33(0.133 \%)$ & $9.99(0.100 \%)$ & $7.27(0.145 \%)$ & $0.43(0.061 \%)$ \\
\hline
\end{tabular}


Table 8.2: Converted standard deviation of confirmation points (subset 2).

\begin{tabular}{|c|c|c|c|c|c|c|}
\hline METHOD & $N F, \mathrm{lbs}$ & $P M$, in-lbs & $S F, \mathrm{lbs}$ & $Y M$, in-lbs & $R M$, in-lbs & $A F, \mathrm{lbs}$ \\
\hline Iterative 1 & $2.93(0.059 \%)$ & $8.78(0.044 \%)$ & $3.43(0.137 \%)$ & $10.28(0.103 \%)$ & $7.33(0.147 \%)$ & $0.45(0.064 \%)$ \\
Iterative 2 & $2.93(0.059 \%)$ & $8.78(0.044 \%)$ & $3.43(0.137 \%)$ & $10.28(0.103 \%)$ & $7.33(0.147 \%)$ & $0.45(0.064 \%)$ \\
Non-Iterative & $2.93(0.059 \%)$ & $8.80(0.044 \%)$ & $3.47(0.139 \%)$ & $10.40(0.104 \%)$ & $7.55(0.151 \%)$ & $0.45(0.064 \%)$ \\
\hline
\end{tabular}

Finally, after comparing values reported in Table 6.1 and 6.2 with corresponding converted values given in Table 8.1 and 8.2, we see that the standard deviations for the three tested methods show good agreement. The predictive capability of all three methods appears to be compatible keeping at the same time in mind that the regression models used by the tested load calculation methods were not identical.

\section{Conclusions}

Data from the machine calibration of a six-component force balance was used to investigate the accuracy of iterative and non-iterative strain-gage balance load calculation methods. Two iterative and one noniterative method were used for the analysis of the calibration data. The balance loads were also processed in both the direct-read and force balance format. In addition, the latest improvements of a regression model optimization process were discussed in some detail as the optimization process was used to select regression models for the analysis of the balance calibration data.

Standard deviations of the load residuals of calibration data points and regression model independent confirmation points were compared after the completion of the regression analysis of the calibration data. In addition, standard deviations obtained in force balance format were transformed to corresponding values in direct-read format so that the influence of the load format on the load prediction accuracy could be investigated.

Overall, all three load calculation methods appear to have compatible load prediction accuracies. In addition, it was noticed that the second iterative method does only converge to a solution if the balance data is processed in force balance format. This observation can be explained by the fact that the iteration equation of the second iterative method implicitly assumes that the primary gage sensitivity of all gages exists. This assumption is violated whenever a force (or moment) balance data set is processed in directread format. The first iterative method, on the other hand, can process calibration data in both direct-read and force balance format as the iteration equation of this method does not depend on the existence of the primary gage sensitivities.

\section{Acknowledgements}

The author would like to thank Tom Volden of Jacobs Technology for his critical and constructive review of the final manuscript of the paper. The work reported in this paper was partially supported by NASA's Aeronautic Test Program and the Wind Tunnel Division at Ames Research Center under contract NNA09DB39C.

\section{References}

${ }^{1}$ AIAA/GTTC Internal Balance Technology Working Group, "Recommended Practice, Calibration and Use of Internal Strain-Gage Balances with Application to Wind Tunnel Testing," AIAA R-091-2003, sponsored and published by the American Institute of Aeronautics and Astronautics, Reston, Virginia, 2003, pp. $18-19$.

${ }^{2}$ Ulbrich, N. and Volden, T., "Strain-Gage Balance Calibration Analysis Using Automatically Selected Math Models," AIAA 2005-4084, paper presented at the 41st AIAA/ASME/SAE/ASEE Joint Propulsion Conference and Exhibit, Tucson, Arizona, July 2005, pp. 11-14.

${ }^{3}$ Ulbrich, N. and Volden, T., "Application of a New Calibration Analysis Process to the MK-III-C Balance," AIAA 2006-0517, paper presented at the 44th AIAA Aerospace Sciences Meeting and Exhibit, Reno, Nevada, January 2006, p. 3, pp. 9-12. 
${ }^{4}$ Ulbrich, N., "Regression Model Optimization for the Analysis of Experimental Data," AIAA 2009-1344, paper presented at the 47th AIAA Aerospace Sciences Meeting and Exhibit, Orlando, Florida, January 2009.

${ }^{5}$ Ulbrich, N., "Optimization of Regression Models of Experimental Data using Confirmation Points," AIAA 2010-0930, paper presented at the 48th AIAA Aerospace Sciences Meeting and Exhibit, Orlando, Florida, January 2010.

${ }^{6}$ Ulbrich, N. and Bader, J., "Analysis of Sting Balance Calibration Data using Optimized Regression Models," AIAA 2009-5372, paper presented at the 45th AIAA/ASME/SAE/ASEE Joint Propulsion Conference \& Exhibit, Denver, Colorado, August 2009, pp. 18-21.

${ }^{7}$ Myers, R. H. and Montgomery, D. C., Response Surface Methodology: Process and Product Optimization Using Designed Experiments, 1st ed., John Wiley \& Sons, Inc., New York, 1995, pp. 24-31. 


\section{Appendix: Identification of Recommended Math Model}

A regression model optimization process is being used at the Balance Calibration Laboratory at NASA Ames Research Center for the automated regression analysis of strain-gage balance calibration data and other multivariate experimental data sets (see Ref. [2], [3], and [4] for a detailed description of the optimization process that uses a candidate math model search algorithm). At the beginning of 2010 an important addition to the regression model optimization process was made. Now, the maximum of the F-Value of Regression of the candidate math models may be used as an alternate identification metric for the selection of the recommended math model after the completion of the candidate math model search.

Key ideas of the candidate math model search algorithm need to be reviewed in order to better understand the advantage of using the maximum of the F-Value of Regression as an alternate identification metric for the selection of the recommended math model. In principle, the candidate math model search algorithm minimizes a search metric during the search. This search metric is the standard deviation of the PRESS residuals of a regression model if no confirmation points are a part of the experimental data set (see Ref. [5] for a discussion of different search metric options). By design, the search metric is a monotonically decreasing function for most data sets if it is plotted versus the number of regression model terms. One of the candidate math models needs to be chosen as the recommended math model after the completion of the search.

Different techniques were implemented over the years in order to select the recommended math model from the final set of candidate math models. Initially, the search algorithm applied a user selected "empirical" threshold in order to identify the recommended math model. This threshold was compared with the difference of the search metric value of two consecutive candidate math models. The recommended math model was picked as soon as the difference was less than the specified threshold (see Ref. [3], p. 3, for a description of this initial selection approach). This initial identification method is no longer used in the algorithm.

A major revision of the search algorithm was made in 2007. At that time more advanced statistical metrics and several search constraints were introduced in the candidate math model search algorithm. This improved version of the algorithm no longer required a user input for the selection of the recommended math model. It was simply chosen to be the candidate math model that had the smallest search metric value while satisfying all search constraints. This identification metric, i.e., the minimum of the search metric, is still being used as the default choice in the current version of the algorithm. It is the primary identification metric for the selction of the recommended math model.

Studies were performed at the beginning of 2010 that compared the F-Value of Regression of the candidate math models with the corresponding plot of the search metric. During these studies an interesting connection between the F-Value of Regression and the search metric was observed:

\section{The F-Value of Regression has a maximum for the candidate math model that gives the "best return on investment" assuming that the number of regression model terms is considered to be the "investment" and the magnitude of the search metric is considered to be the "return."}

It was also observed that the math model identified by the maximum of the F-Value of Regression has fewer terms than the math model that would be chosen by using the primary identification metric discussed in a previous paragraph. Therefore, it was decided to introduce the maximum of the F-Value of Regression of the candidate math models as an alternate identification metric for the selection of the recommended math model as this metric appears to pick a recommended math model that uses the smallest possible set of significant regression model terms.

It still needs to be shown mathematically that the F-Value of Regression of the candidate math models has a maximum if it is plotted versus the number of regression model terms of the candidate math models. The proof starts with the definition of the F-Value of Regression of a regression model of some experimental data. It is defined as follows (from Ref. [7], Eq. (2.21)):

$$
F_{\circ}=\frac{S S_{R} / k}{S S_{E} /(n-k-1)}=\frac{S S_{R}}{S S_{E}} \cdot \frac{n-k-1}{k}
$$

where $S S_{R}$ is the sum of squares due to regression, $S S_{E}$ is the sum of squares due to residual, $n$ is the 
number of data points (observations), and $k$ is the number of terms of the regression model (not counting the intercept). We also know that the total sum of squares, i.e., $S_{y y}$, is the sum of (i) the sum of squares due to regression and (ii) the sum of squares due to residual (see Ref. [7], Eq. (2.20)). Therefore, we can write:

$$
S_{y y}=S S_{R}+S S_{E}
$$

or, after solving Eq. (10a) for $S S_{R}$, we get

$$
S S_{R}=S_{y y}-S S_{E}
$$

Now, after using the right hand side of Eq. (10b) to replace $S S_{R}$ in Eq. (9), we get:

$$
F_{\circ}=\left[\frac{S_{y y}-S S_{E}}{S S_{E}}\right] \cdot\left[\frac{n-k-1}{k}\right]
$$

We also know from the literature that the total sum of squares $\left(S_{y y}\right)$ is only a function of (i) the dependent variables (responses) and (ii) the number of data points. It is defined as (see Ref. [7], p. 29):

$$
S_{y y}=\sum_{i=1}^{n} y_{i}^{2}-\frac{1}{n} \cdot\left(\sum_{i=1}^{n} y_{i}\right)^{2}
$$

where $y_{i}$ is a dependent variable value (response value) at data point $i$. Similarly, we know that the sum of squares due to residual $\left(S S_{E}\right)$ is defined as (see Ref. [7], p. 26):

$$
S S_{E}=\sum_{i=1}^{n}\left(y_{i}-\hat{y}_{i}\right)^{2}
$$

where $\hat{y}_{i}$ is the fitted dependent variable value (response value) at data point $i$. We know from Eq. (11) that the F-Value of Regression of the candidate math models is closely linked to the search metric value because the search metric is the standard deviation of the PRESS residuals of the experimental data set, i.e., a value that closely follows the behavior of the sum of squares due to residual of the fit. Therefore, it is sufficient to look at the behavior of the sum of squares due to residual $\left(S S_{E}\right)$ of the candidate math models instead of looking at the search metric itself.

The proof has to examine the different terms that are used in Eq. (11) to compute the F-Value of Regression. First, the total sum of squares $\left(S_{y y}\right)$ is investigated. From Eq. (10a) we see that $S_{y y}$ is independent of the regression model of the data. It is a constant for the experimental data set. This conclusion can be summarized as follows:

\section{Conclusion 1: $\quad S_{y y} \Longrightarrow$ constant for experimental data set}

The situation is different for the sum of squares due to residual. This value decreases initially and approaches an asymptote as it closely follows the behavior of the search metric that is used during the candidate math model search. Therefore, we can summarize this conclusion using the following statement:

$$
\text { Conclusion 2: } S S_{E} \Longrightarrow \text { decreases and approaches an asymptote as } k \text { increases }
$$

Now, knowing that $S_{y y}$ is a constant (Conclusion 1) and that $S S_{E}$ decreases and approaches an asymptote as the number of regression model terms increases (Conclusion 2), we can make the following statement about the difference of the two values:

$$
\text { Conclusion 3: } \quad S_{y y}-S S_{E} \quad \Longrightarrow \quad \text { increases and approaches an asymptote as } k \text { increases }
$$

Similarly, after computing the inverse of $S S_{E}$ and after considering Conclusion 2, we can say:

$$
\text { Conclusion } 4: \frac{1}{S S_{E}} \Longrightarrow \text { increases and approaches an asymptote as } k \text { increases }
$$


Now, after combining Conclusions 3 and 4, we can make the following statement about the first factor on the right hand side of Eq. (11):

$$
\text { Conclusion 5: }\left[\frac{S_{y y}-S S_{E}}{S S_{E}}\right] \Longrightarrow \text { increases and approaches an asymptote as } k \text { increases }
$$

Similarly, examining the second factor on the right hand side of Eq. (11), we can make the following two statements:

$$
\begin{gathered}
\text { Conclusion } 6: \quad n-k-1 \Longrightarrow \text { decreases as } k \text { increases } \\
\text { Conclusion } 7: \frac{1}{k} \Longrightarrow \text { decreases as } k \text { increases }
\end{gathered}
$$

Therefore, after combining Conclusions 6 and 7, we can make the following statement about the second factor on the right hand side of Eq. (11):

$$
\text { Conclusion 8: }\left[\frac{n-k-1}{k}\right] \Longrightarrow \text { decreases as } k \text { increases }
$$

We see, after applying Conclusion 5 and Conclusion 8 to the definition of the F-Value of Regression given in Eq. (11), that the first factor $\left(S_{y y}-S S_{E}\right) / S S_{E}$ increases initially as $k$ grows. At some point, however, the first factor approaches an asymptote. The second factor $(n-k-1) / k$, on the other hand, decreases monotonically as $k$ grows. Therefore, the $\mathrm{F}$-Value of Regression must have a maximum if plotted as a function of the number of terms of the candidate math models. This statement can be summarized as follows:

\section{Conclusion $9: \quad F_{\circ} \Longrightarrow$ must have a maximum if plotted versus number of terms}

Finally, the above conclusions can be summarized as follows: (i) The regression model of the maximum of the F-Value of Regression may be considered to be the regression model that gives the "best return on investment" assuming that the number of regression coefficients $k$ is considered to be the "investment" and the sum of squares of residual $S S_{E}$ is considered to be the "return." (ii) Therefore, because $S S_{E}$ closely follows the search metric value of the candidate math models, the candidate math model of the maximum of the F-Value of Regression should be chosen as the recommended math model.

Calibration data of the MC60D balance may be used to illustrate the connection between the search metric, the maximum of the F-Value of Regression of the candidate math models, and the recommended math model. Therefore, it was decided to use the non-iterative method in order to obtain a regression model of the axial force as a function of the six gage outputs of the MC60D balance. The optimization of the regression model of the axial force was performed using backward elimination as the search strategy and using the maximum of the F-Value of Regression to select the recommended math model.

Figure 4a shows the search metric of the 28 candidate math models that were identified during the search for an optimized regression model of the axial force. A total number of 279 math models were tested during the search in order to obtain the 28 candidate math models that are shown in Fig. 4a. Only 23 of the 28 candidate math models fulfilled search constraints $2 \& 3$ (these models are marked using enlarged and boldface symbols in Fig. 4a). Figure $4 \mathrm{~b}$ shows the F-Value of Regression of all candidate math models. It can be seen that the F-Value of Regression reaches a maximum for the candidate math model that appears to use a minimum number of significant terms (the blue symbol marks the maximum). This candidate math model should be selected as the recommended math model. 


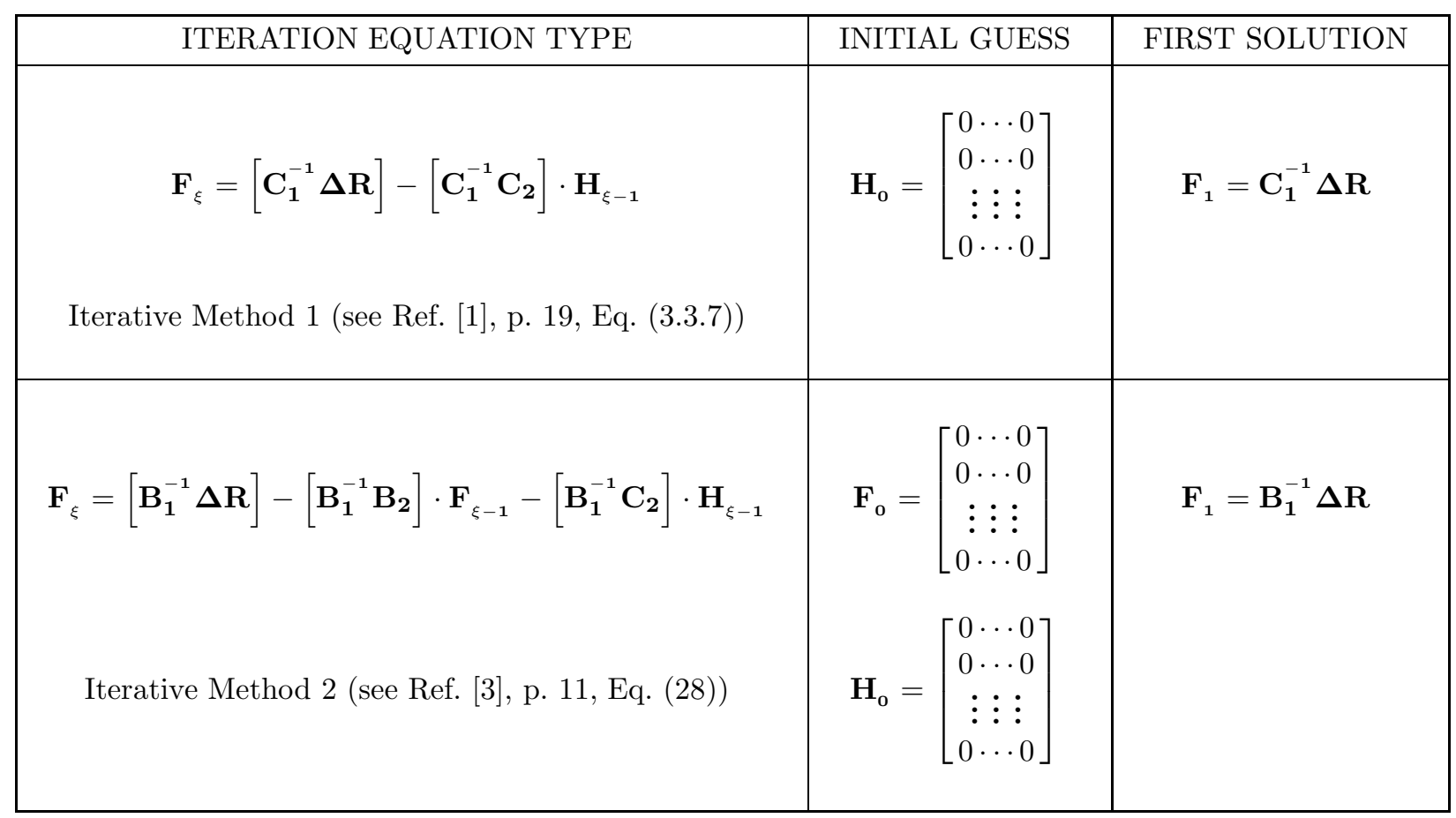

Fig. 1 Definition of iteration equations used by Iterative Method 1 and 2 (see also Ref. [3]).

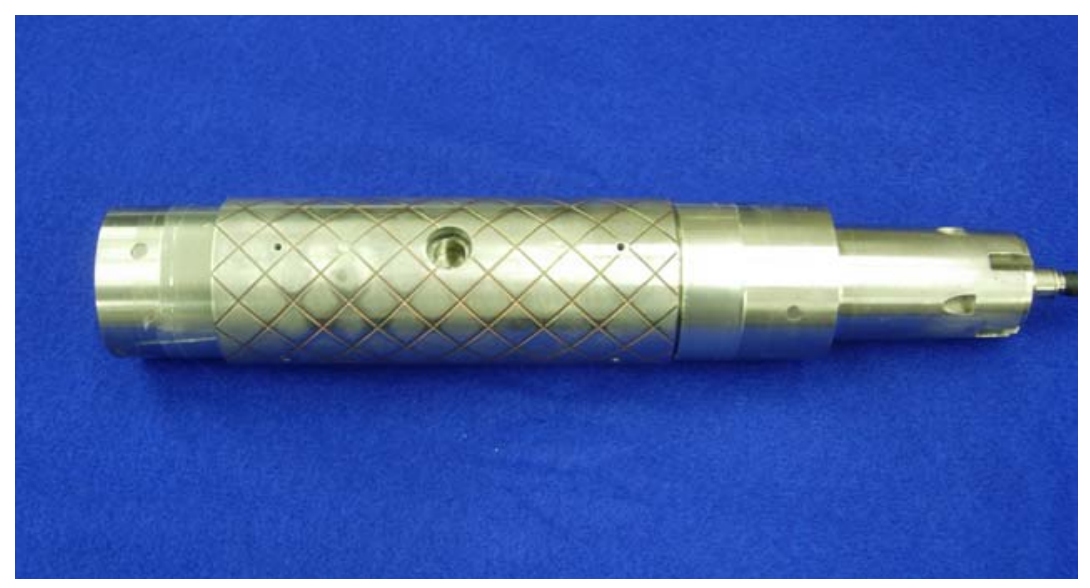

Fig. 2 NASA's MC60D strain-gage balance (photo courtesy of Triumph/Force Measurement Systems). 


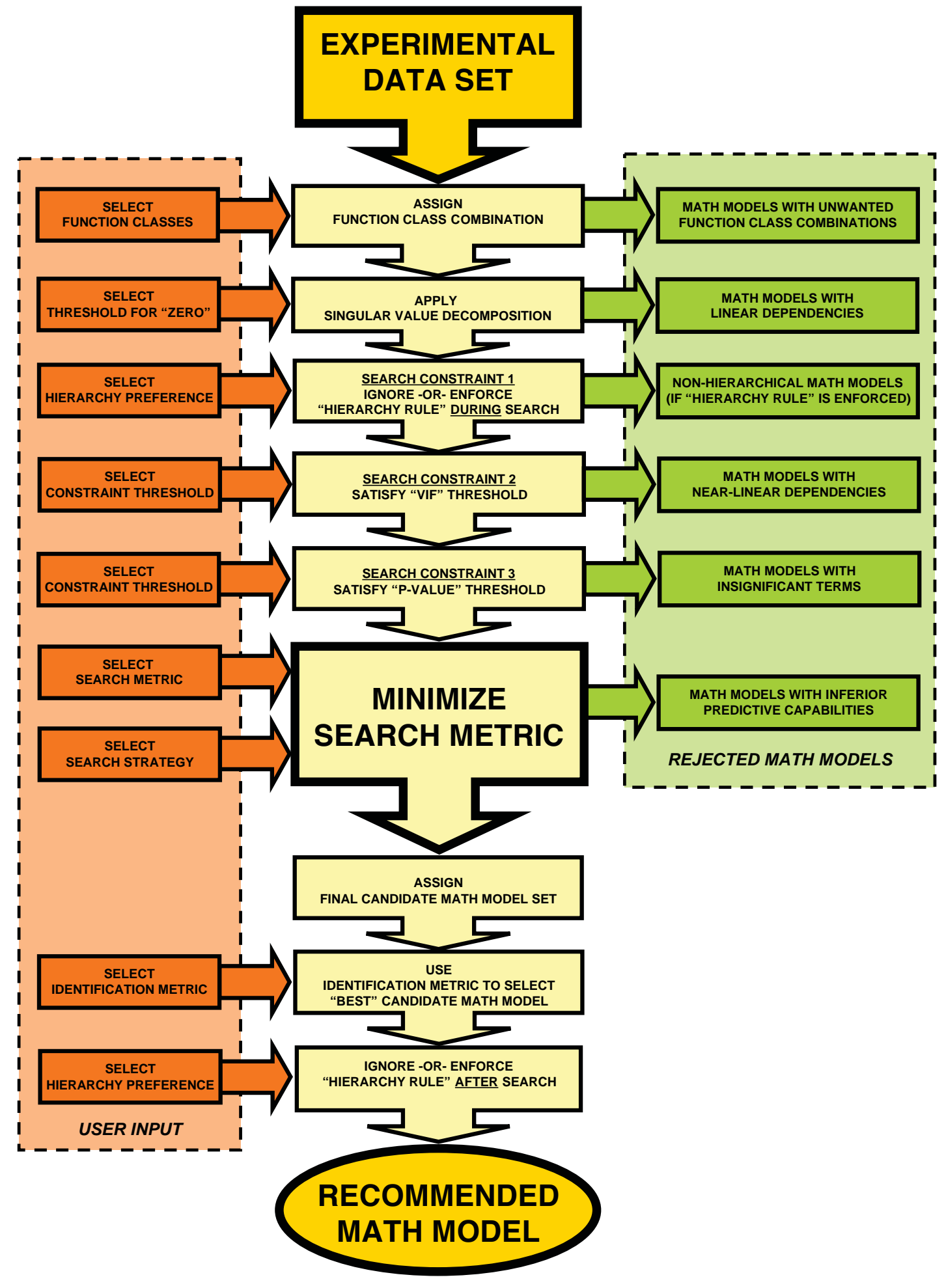

Fig. 3 Candidate math model search algorithm of 2010. 


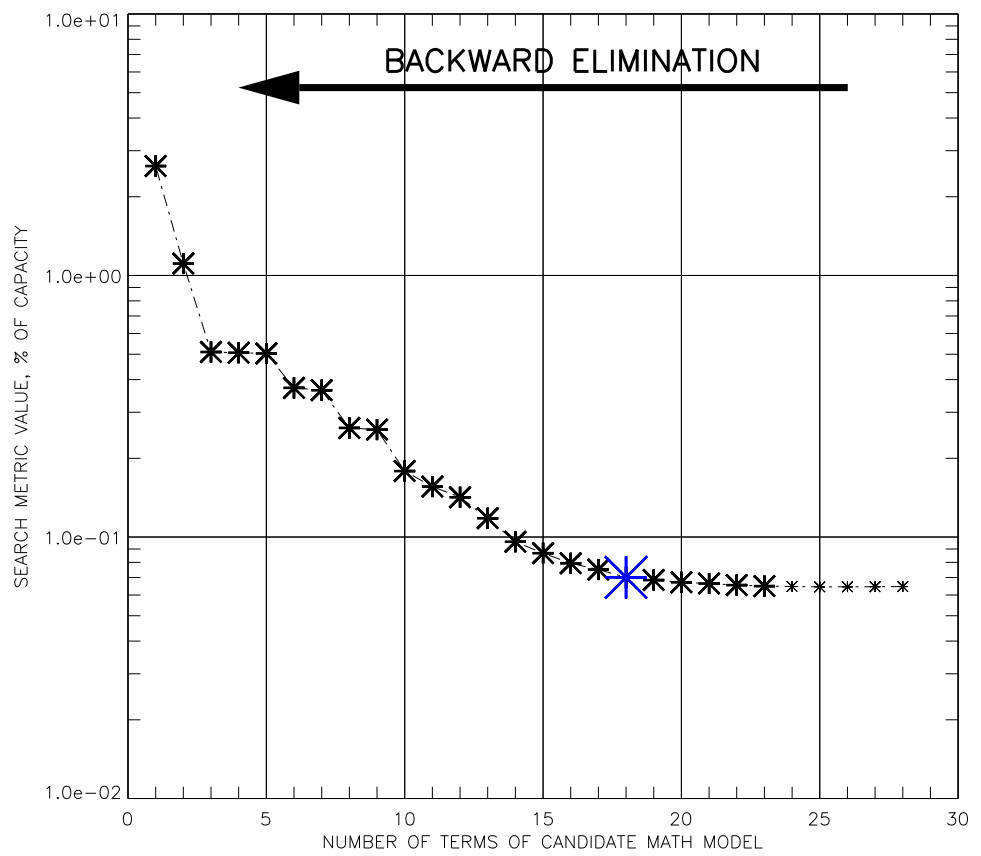

Fig. 4a Search metric versus number of math model terms for candidate math models of axial force. (enlarged symbols $\Longrightarrow$ search constraints $2 \& 3$ fulfilled; blue symbol $\Longrightarrow$ recommended math model)

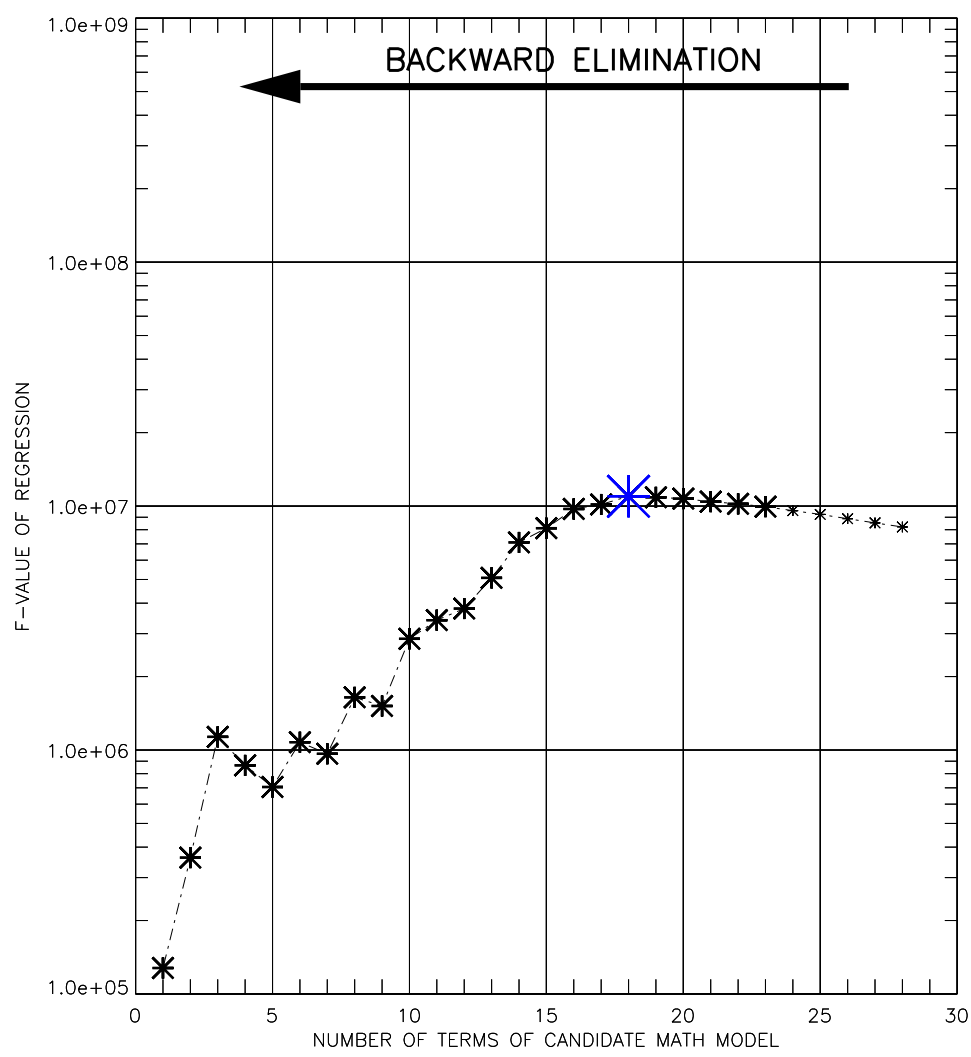

Fig. 4b F-Value of Regression versus number of math model terms for candidate math models of axial force. (enlarged symbols $\Longrightarrow$ search constraints $2 \& 3$ fulfilled; blue symbol $\Longrightarrow$ recommended math model) 